\title{
Awake tracheal intubation in a patient with a supraglottic mass with the Bonfils fibrescope after failed attempts with a flexible fibrescope
}

Geoffrey Liew ${ }^{1}$, MBChB, FRCA, Xin Fang Leong ${ }^{1}$, MBBS, Theodore Wong ${ }^{1}$, MD, FRCPC

\begin{abstract}
Awake intubation with a flexible fibrescope is usually done electively in patients with a known difficult airway. Herein, we describe the case of an elective awake tracheal intubation that was performed on a patient with a large, obstructive supraglottic mass. The intubation was successfully performed using the Bonfils fibrescope after several failed attempts with a flexible fibrescope. This case highlights the usefulness of the Bonfils fibrescope and the limitations of the flexible fibrescope in certain clinical situations.
\end{abstract}

Keywords: Bonfils, difficult airway, flexible fibrescope, oropharyngeal cancer

\section{INTRODUCTION}

Indirect laryngoscopy performed using a flexible fibreoptic laryngoscope remains the gold standard in the management of predicted difficult intubation. ${ }^{(1)}$ Traditionally, awake tracheal intubation is performed using a flexible fibreoptic bronchoscope. The Bonfils Retromolar Intubation Fibrescope (Fig. 1) is a rigid indirect laryngoscope whose use was first described in 1983 for the tracheal intubation of children with Pierre-Robin syndrome. ${ }^{(2)}$ It has been proven to be an effective intubation device for both normal and difficult airways. ${ }^{(3,4)}$ The technique for awake intubation with the Bonfils fibrescope has previously been described. ${ }^{(5-7)}$ We herein describe a successful case of awake tracheal intubation in an anticipated difficult airway using the Bonfils fibrescope, which was used after several unsuccessful attempts with a flexible fibrescope. Informed consent was obtained from the patient prior to the publication of this case report.

\section{CASE REPORT}

A 61-year-old man, who weighed $51 \mathrm{~kg}$ and had a medical history of hyperlipidaemia, presented at our institution for panendoscopy, biopsy of a supraglottic mass and tracheostomy under general anaesthesia. The patient had a three-month history of dysphagia, cough, weight loss and blood-stained saliva, for which he was initially referred to our institution's Ear, Nose and Throat (ENT) Department. Nasendoscopy revealed a large exophytic, supraglottic lesion arising from the left pyriform sinus and extending to the right aryepiglottic fold (Fig. 2). During the preoperative anaesthetic visit, he was found to be mildly stridorous but without respiratory compromise. Airway assessment demonstrated a good mouth opening and unhindered neck movements, with a Mallampati class II score. However, in view of the nasendoscopy findings, the patient was counselled about awake intubation with fibrescopy as well as the contingency

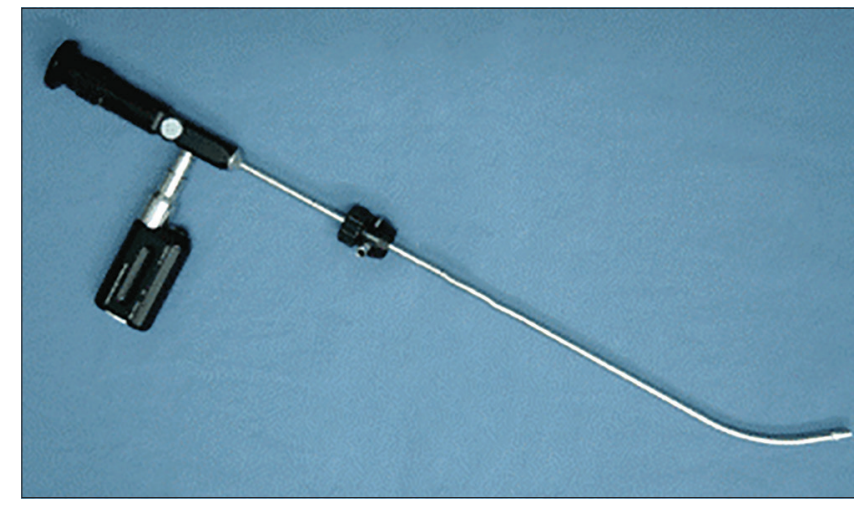

Fig. 1 Photograph shows a Bonfils Retromolar Intubation Fibrescope.
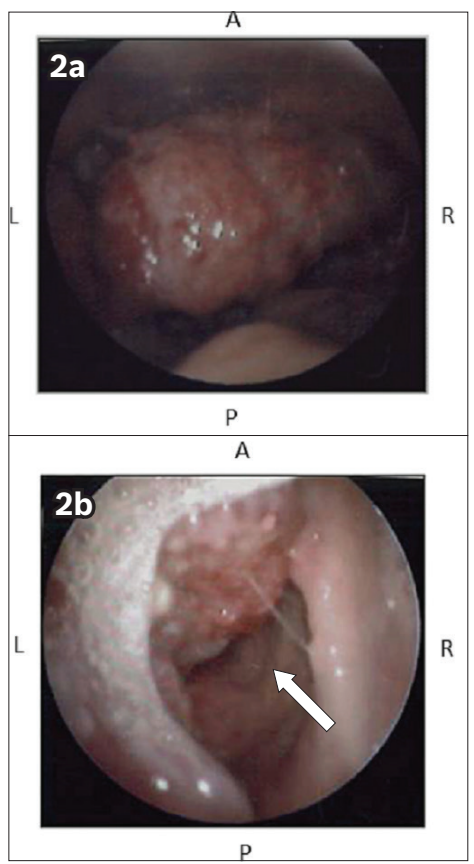

Fig. 2 Nasendoscope images show (a) the supraglottic tumour; and (b) the glottis opening (arrow). 
plan of surgical tracheostomy under local anaesthesia if awake fibreoptic intubation was unsuccessful.

During induction, the patient was placed in a $30^{\circ}$ head-up position. Glycopyrrolate $200 \mu \mathrm{g}$, midazolam $1 \mathrm{mg}$, remifentanil infusion $0.05 \mu \mathrm{g} / \mathrm{kg} /$ minute and supplementary oxygen were administered via nasal prongs. Topicalisation of the airway was performed by spraying $2 \mathrm{~mL}$ of $10 \%$ lidocaine over the mucosa of the mouth, tongue and oropharynx. Size 5.0 microlaryngoscopy tubes were loaded onto an Olympus $4.1 \mathrm{~mm}$ LF-V flexible fibrescope and a Bonfils fibrescope. Initially, the flexible fibrescope was passed via the oral route with the tongue protruded. At the level of the oropharynx, the supraglottic mass, which involved the entire epiglottis, was visualised. It obscured the view of the laryngopharynx and laryngeal inlet except for a 1-2 mm opening in the right lower quadrant of the mass.

Before we attempted to manoeuvre the scope past the mass through the small opening, the mucosa was further topicalised with $2 \mathrm{~mL}$ of $2 \%$ lidocaine via the scope's side port (Fig. 2). After three failed attempts to pass the scope beyond the opening, we proceeded to use the Bonfils fibrescope, advancing it via the right lateral side of the mouth. After spontaneous respiration resulted in the appearance of movement and formation of bubbles lateral to the right aryepiglottic fold, we concluded that the tumour was pressed against and adherent to the inner wall of the right aryepiglottic fold (Fig. 3). Using the Bonfils fibrescope, we managed to part the margin between the tumour and the right aryepiglottic fold, allowing access to the glottic opening. The microlaryngoscopy tube was then railroaded over the Bonfils fibrescope without difficulty. The position of the tube was confirmed, and propofol $50 \mathrm{mg}$ and atracurium $20 \mathrm{mg}$ were administered before the inflation of the cuff; anaesthesia was maintained with desflurane. No immediate complications or trauma from the intubation was observed. Surgical tracheostomy was then performed by the ENT surgeons. Subsequent panendoscopy showed a large supraglottic tumour involving the entire epiglottis, bilateral arytenoids, aryepiglottic folds, vallecula and left pyriform sinus.

\section{DISCUSSION}

The Bonfils fibrescope is a 40-cm-long, straight and rigid fibrescope with an outer diameter of $5.0 \mathrm{~mm}$ and a fixed anterior curvature of $40^{\circ}$ (Fig. 4). The eyepiece, at its proximal end, is used for direct viewing; the fibrescope can also be attached to a video monitor for better visualisation and teaching purposes. As the Bonfils fibrescope is an optical stylet, it allows simultaneous scope visualisation of both the glottis and the endotracheal tube (ETT). The ability to view the ETT when entering the glottic opening reduces the risk of airway trauma. The tube adaptor of the Bonfils fibrescope permits fixation of the ETT, continuous oxygen insufflation and instillation of a local anaesthetic. The Bonfils fibrescope has been demonstrated to be effective for difficult airway management. It is also a useful rescue device in failed direct laryngoscopy. ${ }^{\left({ }^{8}\right)}$ Its slim profile facilitates intubation in patients with reduced mouth openings or with limited or no neck extension. ${ }^{(9-11)}$

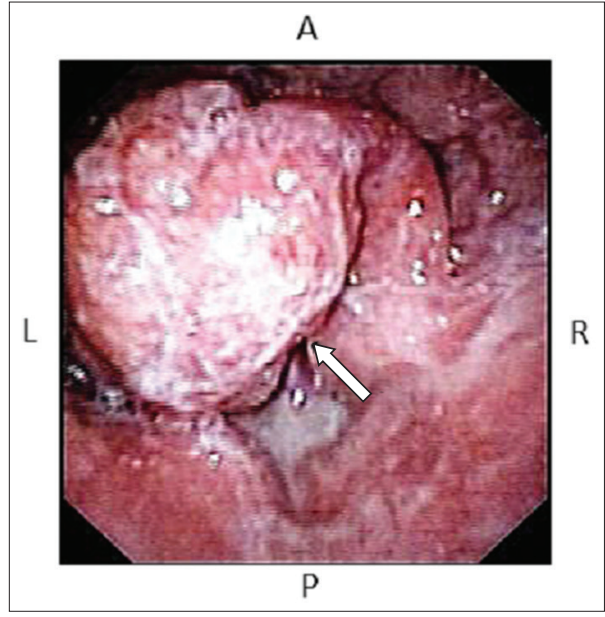

Fig. 3 Flexible fibrescopic image of the supraglottic tumour shows the small opening (arrow) in the right lower quadrant of the mass.

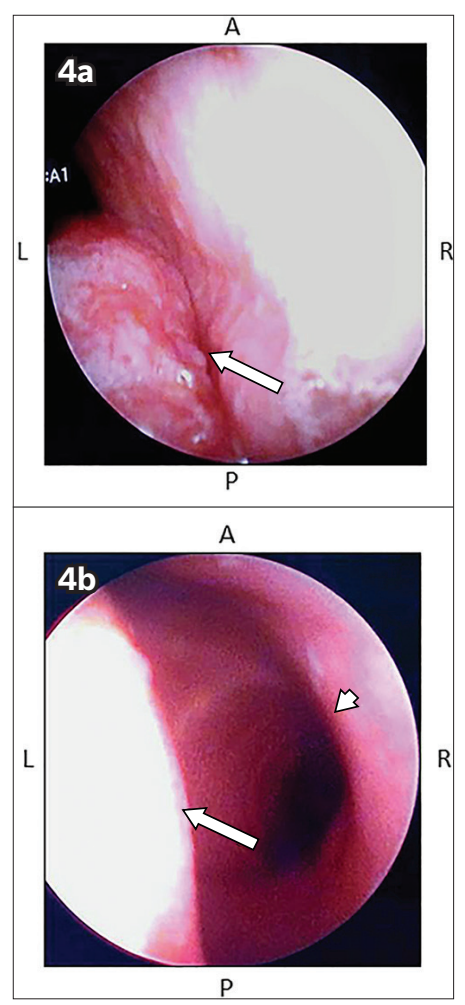

Fig. 4 Bonfils fibrescopic images show (a) the tumour and the small glottis opening (arrow) visualised from the right lateral approach; and (b) visible tracheal rings, the lateral margin of the tumour (arrow) and the aryepiglottic fold (arrowhead), after the Bonfils fibrescope was advanced past the tumour.

Compared to the flexible fibrescope, the Bonfils fibrescope is less expensive, easier to prepare, quicker to clean, and more portable and durable. The Bonfils fibrescope has a rigid body that is able to lift large epiglottises and push away solid tumours to gain access to the glottis. The rigid body allows for easy navigation through the oral cavity, as it is able to displace collapsed soft tissue and obstructions. Railroading the ETT with a Bonfils fibrescope is more straightforward than railroading it with a flexible fibrescope due to the distal loading of the ETT. The Bonfils fibrescope has also been effectively used in awake intubation for patients with difficult airways and/or ENT cancers. Leong and Wong previously reported 
the successful intubation of five patients, who had tumours that obstructed their airways, using the Bonfils fibrescope. ${ }^{(12)}$ The rigidity and small diameter of the Bonfils fibrescope enables the gentle displacement of tumours obstructing the glottic opening, with minimal trauma to the tumour structure. ${ }^{(12)}$ This reduces the risk of distal seeding of the tumour, as well as the bleeding and breaking off of pieces of tumour that could obstruct the airway. In addition, the Bonfils fibrescope allows manipulation with a single hand and therefore provides a more precise translation of hand movement to the tip of the scope. However, unlike the flexible fibrescope, it cannot be used for nasotracheal intubation. Newer models of the Bonfils fibrescope also do not have a working channel for accurate local anaesthetic delivery.

The flexible fibrescope is the primary airway device for managing anticipated difficult airways and awake intubation. As flexible fibrescopes are more readily available than Bonfils fibrescopes in most centres, it is expected that more anaesthetists would be trained in the use of flexible fibrescopes. However, previous surveys have shown that the flexible fibrescopy skills of anaesthetists were fairly limited. ${ }^{(13,14)} \mathrm{A}$ learning curve is expected with the training and teaching of any airway device. The Bonfils fibrescopy is considered to be more difficult to master than other types of videolaryngoscopy. The learning curve may be steep for most clinicians at the start, as its use is not readily intuitive. Some reports suggest that the learning curve improves significantly after performing 20 intubations. ${ }^{(15)}$ However, the learning time for Bonfils orotracheal intubation is likely to be shorter than that for flexible fibreoptic nasotracheal intubation. ${ }^{(16)}$ Nevertheless, the lack of Bonfils fibrescopes in most centres makes training with the device difficult.

The present case report demonstrates that the Bonfils fibrescope can be used effectively for awake tracheal intubation in patients with suspected or known difficult airways once competence with the device has been achieved. It also highlights the limitations of flexible fibrescopy. Bonfils fibrescopy offers advantages over flexible fibrescopy in certain situations, such as when dealing with patients who have large obstructive oropharyngeal tumours (unless nasotracheal intubation is required). In the present case, the flexible fibrescope was unable to negotiate the difficult route around the extensive airway tumour. On the other hand, the Bonfils fibrescope was able to push between the lateral margin of the tumour and the aryepiglottic fold to reach the glottic opening, with minimal trauma to the tumour and airway. When dealing with difficult airways such as the one observed in the present case, extensive airway assessment, careful planning, and a well-thought-out and thorough backup plan are paramount. The backup plan may include the use of flexible fibrescopy, Bonfils fibrescopy and/or awake tracheostomy, as well as expert assistance, airway adjuncts and readily available surgeons. In an elective situation, one should perform an atraumatic and unrushed fibreoptic examination to determine the best approach to intubate the obstructed and distorted airway. Knowledge from preoperative investigations (e.g. nasendoscopy) and careful attention to airway sounds, movements and bubbles should be used to determine the airway structures and their relation to the glottic opening. Since it is not always possible to intubate via flexible fibrescopy, Bonfils fibrescopy should be regarded as an alternative for managing patients with difficult or compromised airways.

\section{REFERENCES}

1. Apfelbaum JL, Hagberg CA, Caplan RA, et al. Practice guidelines for management of the difficult airway: An updated report by the American Society of Anesthesiologists Task Force on Management of the Difficult Airway. Anesthesiology 2013; 118:251-70.

2. Bonfils P. Difficult intubation in Pierre-Robin children, a new method: the retromolar route. Anaesthetist 1983; 32:363-7.

3. Rudolph C, Schlender M. Clinical experiences with fiber optic intubation with the Bonfils intubation fiberscope. Anaesthesiol Reanim 1996; 21:127-30.

4. Halligan M, Charters P. A clinical evaluation of the Bonfils Intubation fibrescope. Anaesthesia 2003; 58:1087-91.

5. Mazères JE, Lefranc A, Cropet C, et al. Evaluation of the Bonfils intubating fibrescope for predicted difficult intubation in awake patients with ear, nose and throat cancer. Eur J Anaesthesiol 2011; 28:646-50.

6. Xue FS, Luo MP, Liao X, He N. Airway topical anesthesia using the Bonfils fiberscope. J Clin Anesth 2009; 21:154-5.

7. Corbanese $U$, Possamai $C$. Awake intubation with Bonfils fibrescope in patients with difficult airway. Eur J Anaesthesiol 2009; 26:837-41.

8. Bein B, Worthmann F, Scholz J, et al. A comparison of the intubating laryngeal mask airway and the Bonfils intubation fibrescope in patients with predicted difficult airways. Anaesthesia 2004; 59:668-74.

9. Byhahn C, Nemetz S, Breitkreutz R, et al. Brief report: tracheal intubation using the Bonfils intubation fibrescope or direct laryngoscopy for patients with a simulated difficult airway. Can J Anaesth 2008; 55:232-7.

10. Rudolph C, Schneider JP, Wallenborn J, Schaffranietz L. Movement of the upper cervical spine during laryngoscopy: a comparison of the Bonfils intubation fibrescope and the Macintosh laryngoscope. Anaesthesia 2005; 60:668-72.

11. Wahlen BM, Gercek E. Three-dimensional cervical spine movement during intubation using the Macintosh and Bullard laryngoscopes, the Bonfils fibrescope and the intubating laryngeal mask airway. Eur J Anaesthesiol 2004; 21:907-13.

12. Leong KW, Wong GLT. The use of the Bonfils retromolar intubation fibrescope in securing the airway in five patients with airway tumours. ASEAN J Anaesth 2011; 12:91-7

13. Ezri T, Szmuk P, Warters RD, Katz J, Hagberg CA. Difficult airway management practice patterns among anesthesiologists practicing in the United States: have we made any progress? J Clin Anesth 2003; 15:418-22.

14. McNarry AF, Dovell T, Dancey FM, Pead ME. Perception in training needs and opportunities in advanced airway skills: a survey of British and Irish trainees. Eur J Anaesthesiol 2007; 24:498-504.

15. Corbanese $U$, Morossi M. The Bonfils intubation fibrescope: clinical evaluation and consideration of the learning curve. Eur J Anaesthesiol 2009; 26:622-4.

16. Smith JE, Jackson AP, Hurdley J, Clifton PJ. Learning curves for fibreoptic nasotracheal intubation when using the endoscopic video camera. Anaesthesia 1997; 52:101-6. 